\title{
On the Lookback Option with Fixed Strike
}

\author{
Yerkin Kitapbayev
}

This version: 11 January 2013

First version: 19 June 2012

Research Report No. 3, 2012, Probability and Statistics Group

School of Mathematics, The University of Manchester 


\title{
On the Lookback Option with Fixed Strike
}

\author{
Yerkin Kitapbayev
}

\begin{abstract}
The lookback option with fixed strike in the case of finite horizon was examined with help of the solution to the optimal stopping problem for a threedimensional Markov process in [1]. The purpose of this paper is to illustrate another derivation of the solution in [1]. The key idea is to use the Girsanov change-of-measure theorem which allows to reduce the three-dimensional optimal stopping problem to a two-dimensional one with a scaling strike. This approach simplifies the discussion and expressions for the arbitrage-free price and the rational exercise boundary. We derive a closed form expression for the value function of the two-dimensional problem in terms of the optimal stopping boundary and show that the optimal stopping boundary itself can be characterised as the unique solution to a nonlinear integral equation. Using these results we obtain the arbitrage-free price and the rational exercise boundary of the option.
\end{abstract}

\section{Introduction}

According to theory of modern finance (see e.g. [13]) the arbitrage-free price of the lookback option with fixed strike coincides with the value function of the optimal stopping problem (2.1) below. In the case of infinite horizon $T$ Shepp and Shiryaev [11] solved the problem when the strike $K$ equals zero (the Russian option) using a two-dimensional Markov setting (stock price and its running maximum). Then in [12] they noticed that this optimal stopping problem can be reduced to a one-dimensional Markov setting by a Girsanov change-of-measure theorem. Pedersen [4] solved the problem (2.1) when $K>0$ in the case of infinite horizon using the maximality principle [5] (for recent extensions to Lévy processes see [3]).

The optimal stopping problems for the maximum processes with finite horizon are inherently three-dimensional (time-process-maximum) and thus analytically more difficult than those with infinite horizon. After applying the change of measure [12] and reducing the problem (2.1) with finite horizon and zero strike (the Russian option) to two dimensions (for a time-space Markov process), the resulting optimal stopping problem was solved by Peskir [7] using the change-ofvariable formula with local time on curves [8]. The optimal stopping boundary was determined as the unique solution of the nonlinear integral equation arising from the formula. Extending this method to the problem (2.1) with finite horizon and non-zero strike (without applying the change of measure), the resulting optimal stopping problem in three dimensions was solved by Gapeev [1] using the change-of-variable formula with local time on surfaces [9].

Mathematics Subject Classification 2010. Primary 91G20,60G40. Secondary 60J60, 35R35, 45G10.

Key words and phrases: American lookback option, arbitrage-free price, fixed strike, optimal stopping, finite horizon, geometric Brownian motion, maximum process, parabolic free-boundary problem, smooth-fit, normal reflection, local time-space calculus, nonlinear integral equation, scaling strike, Markov processes. 
The main purpose of this paper is to illustrate another approach for solving this threedimensional problem using the Girsanov theorem. We show that the arbitrage-free price and optimal stopping set in (2.1) can be expressed by the value function and the optimal stopping boundary of a two-dimensional problem with a scaling strike. Hence we prove all technical conditions in two-dimensional setting. However it is important to emphasize that we first fix strike $K$ and solve the two-dimensional problem, and then to determine the option price and rational exercise boundary we vary the strike, thus the problem inherently remains threedimensional. This method can also be used to derive solution for the British lookback option with fixed strike (cf. Section 7). Another feature of this method is that closed form expression for the value function in (2.1) and nonlinear integral equations for optimal stopping boundary are simpler than in [1]. Dimension of optimal stopping problems often plays a crucial point in finding their solutions, therefore the idea of this approach could be useful in reducing dimension of related optimal stopping problems as well.

In Section 2 we formulate the lookback option with fixed strike in the case of finite horizon. In Section 3 we present reduction of the initial problem to a two-dimensional optimal stopping problem using a change of measure. In Sections 4 and 5 we solve the two-dimensional problem and in Section 6 we apply that solution to the initial problem. In Section 7 we make a conclusion and propose a programme for future research using this approach.

\section{Lookback option with fixed strike}

The arbitrage-free price of the lookback option with fixed strike is given by

$$
V=\sup _{0 \leq \tau \leq T} \mathrm{Ee}^{-r \tau}\left(\max _{0 \leq s \leq \tau} S_{s}-K\right)^{+}
$$

where $\tau$ is a stopping time of the geometric Brownian motion $S$ solving

$$
d S_{t}=r S_{t} d t+\sigma S_{t} d B_{t} \quad\left(S_{0}=s\right)
$$

We recall that $B$ is a standard Brownian motion started at zero, $T>0$ is the expiration date (maturity), $K>0$ is the strike, $r>0$ is the interest rate, and $\sigma$ is the volatility coefficient.

Let us consider the Markovian extension using the structures of processes $\max S$ and $S$, which leads to the following value function

$$
V(t, m, s)=\sup _{0 \leq \tau \leq T-t} \mathrm{E}^{-r \tau}\left(m \vee \max _{0 \leq u \leq \tau} s S_{u}-K\right)^{+}
$$

with $S_{t}=\mathrm{e}^{\sigma W_{t}+\left(r-\frac{\sigma^{2}}{2}\right) t}$ starting at 1 . It is well known that process $M_{t}=\max _{0 \leq s \leq t} S_{s}$ is not Markov, but the pair $(S, M)$ forms a Markov process. Hence this problem is three-dimensional with the Markov process $\left(t, M_{t}, S_{t}\right)_{t \geq 0}$ due to presence of the finite horizon. Since the dimension of the problem is very important, any possibility of reducing the dimension becomes significant. When $K=0$ in [12] and [7] the following reduction was used

$$
\mathrm{E} e^{-r \tau} M_{\tau}=\mathrm{E} e^{-r \tau} S_{\tau} \frac{M_{\tau}}{S_{\tau}}=\widetilde{\mathrm{E}} \frac{M_{\tau}}{S_{\tau}}
$$


where the expectation $\widetilde{\mathrm{E}}$ is taken under a new measure $\widetilde{\mathrm{P}}$ and the process $M / S$ is a onedimensional Markov process under this measure. In the case of non-zero strike one cannot make reduction in the same way straightforwardly. Gapeev [1] solved (2.3) in a three-dimensional setting using the local time-space calculus on surfaces [9]. Current paper illustrates a different approach to the solution of (2.3). Next section explains the reduction of (2.3) to a twodimensional problem with a scaling strike.

\section{Reduction to a two-dimensional problem}

This section will discuss how to reduce problem (2.3) to a two-dimensional problem with a scaling strike.

By the change of measure we have

$$
\begin{aligned}
\mathrm{E}^{-r \tau}\left(m \vee \max _{0 \leq u \leq \tau} s S_{u}-K\right)^{+} & =s \widetilde{\mathrm{E}}\left(\frac{m \vee \max _{0 \leq u \leq \tau} s S_{u}}{s S_{\tau}}-\frac{K}{s S_{\tau}}\right)^{+} \\
& =s \widetilde{\mathrm{E}}\left(\frac{m \vee K \vee \max _{0 \leq u \leq \tau} s S_{u}}{s S_{\tau}}-\frac{K}{s S_{\tau}}\right)
\end{aligned}
$$

where $d \widetilde{\mathrm{P}}=\mathrm{e}^{-r T} S_{T} d \mathrm{P}$ so that $\widehat{B_{t}}=B_{t}-\sigma t$ is a standard Brownian motion under $\widetilde{\mathrm{P}}$ for $0 \leq t \leq T$ and in the second equality we used fact that $(x-y)^{+}=x \vee y-y$ for $x, y \in \mathbb{R}$. The strong solution of (2.2) is given by

$$
S_{t}=s \exp \left(\sigma B_{t}+\left(r-\sigma^{2} / 2\right) t\right)=s \exp \left(\sigma \widehat{B_{t}}+\left(r+\sigma^{2} / 2\right) t\right)
$$

Hence it is easily seen that $\widetilde{\mathrm{E}} \frac{1}{S_{\tau}}=\widetilde{\mathrm{E}} \mathrm{e}^{-r \tau}$ and it follows from (3.1) that we have

$$
\begin{aligned}
\mathrm{Ee}^{-r \tau}\left(m \vee \max _{0 \leq u \leq \tau} s S_{u}-K\right)^{+} & =s \widetilde{\mathrm{E}}\left(\frac{\frac{m \vee K}{s} \vee M_{\tau}}{S_{\tau}}-\frac{K}{s} \mathrm{e}^{-r \tau}\right) \\
& =s \widetilde{\mathrm{E}}\left(\frac{\frac{m \vee K}{s} \vee M_{\tau}}{S_{\tau}}-\frac{K e^{r t}}{s} \mathrm{e}^{-r(t+\tau)}\right)
\end{aligned}
$$

where $M_{t}=\max _{0 \leq u \leq t} S_{u}$. This motivates us to fix $\widetilde{K}=K e^{r t} / s$ and consider the following two-dimensional (time-space) optimal stopping problem:

$$
W(t, x)=W(t, x ; \widetilde{K})=\sup _{0 \leq \tau \leq T-t} \widetilde{\mathrm{E}}\left(X_{\tau}^{x}-\widetilde{K} \mathrm{e}^{-r(t+\tau)}\right)
$$

where $X_{t}^{x}=\frac{x \vee M_{t}}{S_{t}}$ is a Markov process. By Ito's formula one finds that

$$
d X_{t}=-r X_{t} d t+\sigma X_{t} d \widetilde{B}_{t}+d R_{t}
$$

under $\widetilde{\mathrm{P}}$ where $\widetilde{B}=-\widehat{B}$ is a standard Brownian motion, and we set

$$
R_{t}=\int_{0}^{t} I\left(X_{s}=1\right) \frac{d M_{s}}{S_{s}} .
$$


It is clear from (3.3) that the initial value (2.3) can be expressed as

$$
V(t, m, s)=s W\left(t, \frac{m \vee K}{s} ; \frac{K \mathrm{e}^{r t}}{s}\right)
$$

and the optimal stopping set in (2.3) is given by

$$
D=\left\{(t, m, s): s W\left(t, \frac{m \vee K}{s} ; \frac{K \mathrm{e}^{r t}}{s}\right)=(m-K)^{+}\right\}
$$

In the next section we solve the two-dimensional problem (3.4).

\section{The two-dimensional problem}

Let us consider the optimal stopping problem

$$
W(t, x)=\sup _{0 \leq \tau \leq T-t} \widetilde{\mathrm{E}} G\left(t+\tau, X_{\tau}^{x}\right)
$$

where the process $X$ from (3.5) with $X_{0}^{x}=x$ under $\widetilde{\mathrm{P}}, \quad 0 \leq t \leq T, \quad x \geq 1$ and the gain function is given by $G(t, x)=x-K \mathrm{e}^{-r t}$. This section parallels the derivation of the solution [7] when $G(t, x)=x$ for $x \geq 1$ and presents needed modifications since in (4.1) the gain function depends on time in a nonlinear way.

Standard Markovian arguments (see e.g. [10]) indicate that $W$ solves the following freeboundary problem:

$$
\begin{array}{ll}
W_{t}+\mathbb{L}_{X} W=0 & \text { in } C \\
W(t, x)=G(t, x) & \text { for } x=b(t) \\
W_{x}(t, x)=1 & \text { for } x=b(t) \\
W_{x}(t, 1+)=0 & \text { (normal reflection) } \\
W(t, x)>G(t, x) & \text { in } C \\
W(t, x)=G(t, x) & \text { in } D
\end{array}
$$

where the continuation set $C$ and the stopping set $D$ are defined by

$$
\begin{aligned}
& C=\{(t, x) \in[0, T) \times[1, \infty): x<b(t)\} \\
& D=\{(t, x) \in[0, T) \times[1, \infty): x \geq b(t)\}
\end{aligned}
$$

and $b:[0, T] \rightarrow R$ is the unknown optimal stopping boundary, i.e the stopping time

$$
\tau_{b}=\inf \left\{0 \leq s \leq T-t: X_{s}^{x} \geq b(t+s)\right\}
$$

is optimal in the problem (4.1).

Our main aim is to follow the train of thought where $W$ is first expressed in terms of $b$, and $b$ itself is shown to satisfy a nonlinear integral equation. We will moreover see that the nonlinear equation derived for $b$ cannot have other solutions. (We also note that in the Section 
6 we will consider the value function $W(t, x)=W(t, x ; K)$ and the optimal stopping boundary $b(t)=b(t ; K)$ as the functions of strike $K$ as well.) Below we will use the following functions:

$$
\begin{gathered}
F(t, x)=\widetilde{\mathrm{E}}\left(X_{t}^{x}\right) \\
H(t, u, x, y)=\widetilde{\mathrm{E}}_{t, x}\left(G\left(u, X_{u}\right) I\left(X_{u} \geq y\right)\right)=\int_{y}^{\infty}\left(z-K \mathrm{e}^{-r u}\right) f(u-t, x, z) d z
\end{gathered}
$$

for $t \in[0, T], x \geq 1, u \in(t, T], y \geq 1$, where $z \mapsto f(u-t, x, z)$ is the probability density function of $X_{u-t}^{x}$ under $\widetilde{\mathrm{P}}$ and $\widetilde{\mathrm{P}}_{t, x}(\cdot)=\widetilde{\mathrm{P}}\left(\cdot \mid X_{t}=x\right)$.

The main result of present section may now be stated as follows.

Theorem 4.1. The optimal stopping boundary in the problem (4.1) can be characterised as the unique continuous decreasing solution $b:[0, T] \rightarrow \mathbb{R}$ of the nonlinear integral equation

$$
b(t)=K\left(e^{-r t}-e^{-r T}\right)+F(T-t, b(t))+r \int_{t}^{T} H(t, u, b(t), b(u)) d u
$$

satisfying $b(t)>\left(K e^{-r t} \vee 1\right)$ for $0<t<T$. The solution $b$ satisfies $b(T-)=\left(K e^{-r t} \vee 1\right)$ and the stopping time $\tau_{b}$ from (4.10) is optimal in (4.1) (see Figure 1).

The value function (4.1) admits the following representation:

$$
W(t, x)=-K e^{-r T}+F(T-t, x)+r \int_{t}^{T} H(t, u, x, b(u)) d u
$$

for all $(t, x) \in[0, T] \times[1, \infty)$.

Proof. The proof will be carried out in several steps. We start by stating some general remarks.

We see that $W$ admits the following representation:

$$
W(t, x)=\sup _{0 \leq \tau \leq T-t} \widetilde{\mathrm{E}}\left(\frac{\left(x-M_{\tau}\right)^{+}+M_{\tau}}{S_{\tau}}-K \mathrm{e}^{-r(t+\tau)}\right)
$$

for $(t, x) \in[0, T] \times[1, \infty)$. It follows that

$$
x \mapsto W(t, x) \text { is increasing and convex on }[1, \infty)
$$

for each $t \geq 0$ fixed.

1. We show that $W:[0, T] \times[1, \infty) \rightarrow \mathbb{R}$ is continuous. For this, using $\sup (f)-\sup (g) \leq$ $\sup (f-g)$ and $(y-z)^{+}-(x-z)^{+} \leq(y-x)^{+}$for $x, y, z \in \mathbb{R}$, it follows that

$$
W(t, y)-W(t, x) \leq(y-x) \sup _{0 \leq \tau \leq T-t} \widetilde{\mathrm{E}}\left(\frac{1}{S_{\tau}}\right) \leq y-x
$$




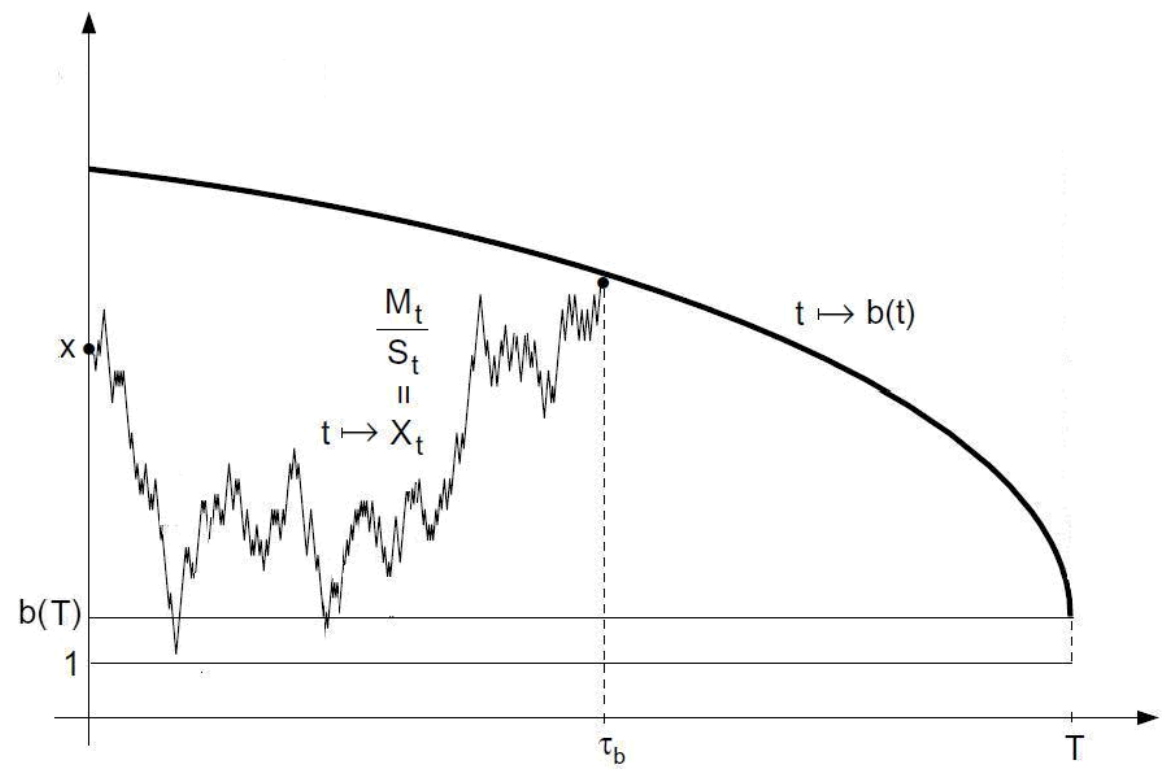

Figure 1. A computer drawing of the optimal stopping boundary $b$ for the problem (4.1) in the case $K=1.5, T=1, r=0.1$ with the boundary condition $b(T)=K \mathrm{e}^{-r T}>1$.

for $1 \leq x<y$ and all $t \geq 0$. From (4.16) and (4.17) we see that $x \mapsto W(t, x)$ is continuous uniformly over $t \in[0, T]$. Thus to prove that $W$ is continuous on $[0, T] \times[1, \infty)$ it is enough to show that $t \mapsto W(t, x)$ is continuous on $[0, T]$ for each $x \geq 1$ given and fixed. For this, take any $t_{1}<t_{2}$ in $[0, T]$ and $\varepsilon>0$, and let $\tau_{1}^{\varepsilon}$ be a stopping time such that $\widetilde{\mathrm{E}}\left(X_{\tau_{1}^{\varepsilon}}^{x}-K \mathrm{e}^{-r\left(t_{1}+\tau_{1}^{\varepsilon}\right)}\right) \geq W\left(t_{1}, x\right)-\varepsilon$. Setting $\tau_{2}^{\varepsilon}=\tau_{1}^{\varepsilon} \wedge\left(T-t_{2}\right)$ we see that $W\left(t_{2}, x\right) \geq$ $\widetilde{\mathrm{E}}\left(X_{\tau_{2}^{\varepsilon}}^{x}-K \mathrm{e}^{-r\left(t_{2}+\tau_{2}^{\varepsilon}\right)}\right)$. Hence, we get

$$
0 \leq\left|W\left(t_{1}, x\right)-W\left(t_{2}, x\right)\right| \leq\left|\widetilde{\mathrm{E}}\left(X_{\tau_{1}^{\varepsilon}}^{x}-K \mathrm{e}^{-r\left(t_{1}+\tau_{1}^{\varepsilon}\right)}-X_{\tau_{2}^{\varepsilon}}^{x}+K \mathrm{e}^{-r\left(t_{2}+\tau_{2}^{\varepsilon}\right)}\right)\right|+\varepsilon .
$$

Letting first $t_{2}-t_{1} \rightarrow 0$ using $\tau_{1}^{\varepsilon}-\tau_{2}^{\varepsilon} \rightarrow 0$ and then $\varepsilon \rightarrow 0$ we see that $\left|W\left(t_{1}, x\right)-W\left(t_{2}, x\right)\right| \rightarrow$ 0 by dominated convergence. This shows that $t \mapsto W(t, x)$ is continuous on $[0, T]$, and the proof of the initial claim is complete.

Introduce the continuation set $C=\{(t, x) \in[0, T] \times[1, \infty): V(t, x)>G(t, x)\}$ and the stopping set $D=\{(t, x) \in[0, T] \times[1, \infty): V(t, x)=G(t, x)\}$. Since $V$ and $G$ are continuous, we see that $C$ is open and $D$ is closed in $[0, T] \times[1, \infty)$. Standard arguments based on the strong Markov property (see [10]) show that the first hitting time $\tau_{D}=\inf \left\{0 \leq s \leq T-t:\left(t+s, X_{s}^{x}\right) \in D\right\}$ is optimal in (4.1).

2. We show that the continuation set $C$ just defined is given by (4.8) for some function $b:[0, T) \rightarrow(1, \infty)$. It follows in particular that the stopping set coincides with the set $D$ in (4.9) as claimed. To verify the initial claim, note that by Ito's formula and (3.5) we have

$$
X_{s}^{x}=x-r \int_{0}^{s} X_{u}^{x} d u+\int_{0}^{s} \frac{d M_{u}}{S_{u}}+N_{s}
$$


where $N_{s}=\sigma \int_{0}^{s} X_{u}^{x} d \widetilde{B}_{u}$ is a martingale for $0 \leq s \leq T$. We will first show that $(t, x) \in C$ implies that $(t, y) \in C$ for $x>y \geq 1$ be given and fixed. For this, let $\tau_{*}=\tau_{*}(t, x)$ denote the optimal stopping time for $W(t, x)$. Using (4.19) and the optimal sampling theorem, we find

$$
\begin{aligned}
W(t, y)-y+K \mathrm{e}^{-r t} & \geq \widetilde{\mathrm{E}}\left[X_{\tau_{*}}^{y}-K \mathrm{e}^{-r\left(t+\tau_{*}\right)}\right]-y+K \mathrm{e}^{-r t} \\
& =-r \widetilde{\mathrm{E}} \int_{0}^{\tau_{*}} X_{u}^{y} d u+\widetilde{\mathrm{E}} \int_{0}^{\tau_{*}} \frac{d M_{u}}{S_{u}}-K \widetilde{\mathrm{E}} \mathrm{e}^{-r\left(t+\tau_{*}\right)}+K \mathrm{e}^{-r t} \\
& \geq-r \widetilde{\mathrm{E}} \int_{0}^{\tau_{*}} X_{u}^{x} d u+\widetilde{\mathrm{E}} \int_{0}^{\tau_{*}} \frac{d M_{u}}{S_{u}}-K \widetilde{\mathrm{E}} \mathrm{e}^{-r\left(t+\tau_{*}\right)}+K \mathrm{e}^{-r t} \\
& =\widetilde{\mathrm{E}}\left[X_{\tau_{*}}^{x}-K \mathrm{e}^{-r\left(t+\tau_{*}\right)}\right]-x+K \mathrm{e}^{-r t} \\
& =W(t, x)-x+K \mathrm{e}^{-r t}>0
\end{aligned}
$$

proving the claim. The fact just proved establishes the existence of a function $b:[0, T] \rightarrow[1, \infty]$ such that the continuation set $C$ is given by (4.8) above.

To gain a deeper insight into the solution, let us apply Ito's formula for $G$ using (4.2),(4.5) and that $G_{t}+\mathbb{L}_{X} G=-r G$ :

$$
G\left(t+s, X_{s}^{x}\right)=G(t, x)-r \int_{0}^{s} G\left(t+u, X_{u}^{x}\right) d u+N_{s}+\int_{0}^{s} d R_{u} .
$$

Thus the optional sampling theorem yield

$$
\widetilde{\mathrm{E}} G\left(t+\tau, X_{\tau}^{x}\right)=G(t, x)-r \widetilde{\mathrm{E}} \int_{0}^{\tau} G\left(t+u, X_{u}^{x}\right) d u+\widetilde{\mathrm{E}} \int_{0}^{\tau} d R_{u}
$$

for all stopping times $\tau$ of $X$ with values in $[0, T-t]$ with $t \in[0, T)$ and $x \geq 1$ given and fixed.

It can be seen from (4.21) and the structure of $G$ that no point $(t, x)$ in $[0, T) \times[1, \infty)$ with $x<\left(K \mathrm{e}^{-r t} \vee 1\right)$ is a stopping point (for this one can make use of the first exit time from a sufficiently small time-space ball centred at the point). Likewise, it is also clear and can be verified that if $x>\left(K \mathrm{e}^{-r t} \vee 1\right)$ and $t<T$ is sufficiently close to $T$ then it is optimal to stop immediately (since the gain obtained from being below $G$ cannot offset the cost of getting there due to the lack of time). This shows that the optimal stopping boundary $b$ satisfies $b(T)=\left(K \mathrm{e}^{-r T} \vee 1\right)$. It is also clear and can be verified that if the initial point $x \geq 1$ of the process $X$ is sufficiently large then it is optimal to stop immediately (since the gain obtained from being below $G$ cannot offset the cost of getting there due to the shortage of time). This shows that the optimal stopping boundary $b$ is finite valued.

In [7] it was shown that the optimal stopping boundary $b(t ; 0)>1$ for $t \in[0, T)$ when $K=0$. It is easily seen that $b(t ; K) \geq b(t ; 0)$ for $t \in[0, T)$ and $K>0$. Thus we have that $b(t)>1$ for every $t \in[0, T)$.

3. We show that the smooth-fit condition (4.4) holds. For this, let $t \in[0, T)$ be given and fixed and set $x=b(t)$. We know that $x>1$ so that there exists $\varepsilon>0$ such that $x-\varepsilon>1$ too. Since $W(t, x)=G(t, x)$ and $W(t, x-\varepsilon)>G(t, x-\varepsilon)$, we have:

$$
\frac{W(t, x)-W(t, x-\varepsilon)}{\varepsilon} \leq \frac{G(t, x)-G(t, x-\varepsilon)}{\varepsilon} \leq 1
$$


Then, let $\tau_{\varepsilon}=\tau_{\varepsilon}^{*}(t, x-\varepsilon)$ denote the optimal stopping time for $W(t, x-\varepsilon)$. We have

$$
\begin{aligned}
\frac{W(t, x)}{}-W(t, x-\varepsilon) \\
\quad \geq \frac{1}{\varepsilon} \widetilde{\mathrm{E}}\left(\frac{\left(x-M_{\tau_{\varepsilon}}\right)^{+}+M_{\tau_{\varepsilon}}}{S_{\tau_{\varepsilon}}}-\frac{\left(x-\varepsilon-M_{\tau_{\varepsilon}}\right)^{+}+M_{\tau_{\varepsilon}}}{S_{\tau_{\varepsilon}}}\right) \\
=\frac{1}{\varepsilon} \widetilde{\mathrm{E}}\left(\frac{\left(x-M_{\tau_{\varepsilon}}\right)^{+}}{S_{\tau_{\varepsilon}}}-\frac{\left(x-\varepsilon-M_{\tau_{\varepsilon}}\right)^{+}}{S_{\tau_{\varepsilon}}}\right) \\
\geq \frac{1}{\varepsilon} \widetilde{\mathrm{E}}\left(\frac{1}{S_{\tau_{\varepsilon}}}\left(\left(x-M_{\tau_{\varepsilon}}\right)^{+}-\left(x-\varepsilon-M_{\tau_{\varepsilon}}\right)^{+}\right) I\left(M_{\tau_{\varepsilon}} \leq x-\varepsilon\right)\right) \\
=\widetilde{\mathrm{E}}\left(\frac{1}{S_{\tau_{\varepsilon}}} I\left(M_{\tau_{\varepsilon}} \leq x-\varepsilon\right)\right) \rightarrow 1
\end{aligned}
$$

as $\varepsilon \downarrow 0$ by bounded convergence, since $\tau_{\varepsilon} \rightarrow 0$ so that $M_{\tau_{\varepsilon}} \rightarrow 1$ with $1<x-\varepsilon$ and likewise $S_{\tau_{\varepsilon}} \rightarrow 1$. It thus follows from (4.16),(4.22) and (4.23) that $W_{x}^{-}(t, x) \geq 1$ and $W_{x}^{-}(t, x) \leq 1$. Thus we have that $W_{x}^{-}(t, x)=1$. Since $W(t, y)=G(t, y)$ for $y>x$, it is clear that $W_{x}^{+}(t, x)=1$. We may thus conclude that $y \mapsto W(t, y)$ is $C^{1}$ at $b(t)$ and $W_{x}(t, b(t))=1$ as stated in (4.4).

4. We show that inequality is satisfied:

$$
W_{t}(t, x) \leq G_{t}(t, x)
$$

for all $0<t<T$ and $x \geq 1$.

To prove (4.24) fix $0<t<t+h<T$ and $x \geq 1$. Let $\tau$ be the optimal stopping time for $W(t+h, x)$. Since $\tau \in[0, T-t-h] \subseteq[0, T-t]$ we see that $W(t, x) \geq \widetilde{\mathrm{E}}\left(X_{\tau}^{x}-K \mathrm{e}^{-r(t+\tau)}\right)$ and we get:

$$
\begin{aligned}
W(t+ & h, x)-W(t, x)-(G(t+h, x)-G(t, x)) \\
& \leq \widetilde{\mathrm{E}}\left(-K e^{-r(t+h+\tau)}+K e^{-r(t+\tau)}\right)+K e^{-r(t+h)}-K e^{-r t} \\
& =K e^{-r t} \widetilde{\mathrm{E}}\left(-e^{-r(h+\tau)}+e^{-r \tau}+e^{-r h}-1\right) \\
& =K e^{-r t} \widetilde{\mathrm{E}}\left(e^{-r h}\left(-e^{-r \tau}+1\right)+e^{-r \tau}-1\right) \\
& =K e^{-r t} \widetilde{\mathrm{E}}\left(e^{-r h}-1\right)\left(-e^{-r \tau}+1\right) \leq 0 .
\end{aligned}
$$

Dividing initial expression in (4.25) by $h$ and letting $h \downarrow 0$ we obtain (4.24) for all $(t, x)$.

5. We show that $b$ is decreasing on $[0, T]$. This is an immediate consequence of (4.25). Indeed, if $\left(t_{2}, x\right)$ belongs to $C$ and $t_{1}$ from $(0, T)$ satisfies $t_{1}<t_{2}$, then by (4.25) we have that $V\left(t_{1}, x\right)-G\left(t_{1}, x\right) \geq V\left(t_{2}, x\right)-G\left(t_{2}, x\right)>0$ so that $\left(t_{1}, x\right)$ must belong to $C$. It follows that $b$ is decreasing thus proving the claim.

6. We show that $b$ is continuous. Note that the same proof also shows that $b(T-)=$ $\left(K \mathrm{e}^{-r T} \vee 1\right)$ as already established above.

Since the stopping set equals $D=\{(t, x) \in[0, T) \times[1, \infty): V(t, x)=G(t, x)\}$ and $b$ is decreasing, it is easily seen that $b$ is right-continuous on $[0, T)$. 
Then note that since the supremum in (4.1) is attained at the first exit time $\tau_{b}$ from the open set $C$, standard arguments based on the strong Markov property (cf. [10]) imply that $W$ is $C^{1,2}$ on $C$ and satisfies (4.2). Suppose that there exists $t \in(0, T)$ such that $b(t-)>b(t)$ and fix any $x \in[b(t), b(t-))$. Note that by (4.4) we have:

$$
W(s, x)-x+K \mathrm{e}^{-r s}=\int_{x}^{b(s)} \int_{y}^{b(s)} W_{x x}(s, z) d z d y
$$

for each $s<t$. If $W_{x x} \geq c$ on $P=\{(u, y) \in C \mid s \leq u<t$ and $x \leq y<b(u)\}$ for some $c>0$ (for all $s<t$ close enough to $t$ and some $x<b(t-)$ close enough to $b(t-)$ ) then by letting $s \uparrow t$ in (4.26) we get:

$$
W(t, x)-x+K \mathrm{e}^{-r t} \geq c \frac{(b(t-)-x)^{2}}{2}>0
$$

contradicting the fact that $(t, x)$ belongs to $D$ and thus is an optimal stopping point. Hence the proof reduces to showing that $W_{x x} \geq c$ on small enough $P$ for some $c>0$.

To derive the latter fact we may first note from (4.2) upon using (4.24) that $\frac{\sigma^{2}}{2} x^{2} W_{x x}=$ $-W_{t}+r x W_{x} \geq-r K \mathrm{e}^{-r t}+r x W_{x}$ in $C$. Suppose now that for each $\delta>0$ there is $s<t$ close enough to $t$ and there is $x<b(t-)$ close enough to $b(t-)$ such that $W_{x}(u, y) \geq 1-\delta$ for all $(u, y) \in P$ (where we recall that $1=G_{x}(u, y)$ for all $(u, y) \in P$ ). Then from the previous inequality we find that $W_{x x}(u, y) \geq\left(2 /\left(\sigma^{2} y^{2}\right)\right)\left(r\left(y-K \mathrm{e}^{-r u}\right)-r y \delta\right) \geq c>0$ for $\delta>0$ small enough since $y>K \mathrm{e}^{-r u}$ and for all $(u, y) \in P$. Hence the proof reduces to showing that $W_{x}(u, y) \geq 1-\delta$ for all $(u, y) \in P$ with $P$ small enough when $\delta>0$ is given and fixed.

To derive latter inequality we can make use of the estimate (4.23) to conclude that

$$
\frac{W(u, y)-W(u, y-\varepsilon)}{\varepsilon} \geq \widetilde{\mathrm{E}}\left(\frac{1}{S_{\sigma_{\varepsilon}}} I\left(M_{\sigma_{\varepsilon}} \leq x-\varepsilon\right)\right)
$$

where $\sigma_{\varepsilon}=\inf \left\{0 \leq v \leq T-u: X_{v}^{y-\varepsilon}=b(u+v)\right\}$ and $M_{t}=\max _{0 \leq s \leq t} S_{s}$. Using the fact that $b$ is decreasing and letting $\varepsilon \rightarrow 0$ in (4.28) we get

$$
W_{x}(u, y) \geq \widetilde{\mathrm{E}}\left(\frac{1}{S_{\sigma}} I\left(M_{\sigma} \leq x\right)\right)
$$

for all $(u, y) \in P$ where $\sigma=\inf \left\{0 \leq v \leq T-s: X_{v}^{x}=b(s+v)\right\}$. Since by regularity of $X$ we find that $\sigma \downarrow 0 \quad \widetilde{\mathrm{P}}-$ a.s. as $s \uparrow t$ and $x \downarrow b(t-)$, it follows from (4.29) that

$$
W_{x}(u, y) \geq 1-\delta
$$

for all $s<t$ close enough to $t$ and some $x>b(t-)$ close enough to $b(t-)$. This completes the proof of the claim.

7. We show that the normal reflection condition (4.5) holds. For this, note first that since $x \mapsto W(t, x)$ is increasing (and convex) on $[1, \infty)$ it follows that $W_{x}(t, 1+) \geq 0$ for all $t \in[0, T)$. Suppose that there exists $t \in[0, T)$ such that $W_{x}(t, 1+)>0$. Recalling that $W$ is $C^{1,2}$ on $C$ so that $t \mapsto W_{x}(t, 1+)$ is continuous on $[0, T)$, we see that there exists $\delta>0$ 
such that $W_{x}(s, 1+) \geq \varepsilon>0$ for all $s \in[t, t+\delta]$ with $t+\delta<T$. Setting $\tau_{\delta}=\tau_{b} \wedge \delta$ it follows by Ito's formula that

$$
\widetilde{\mathrm{E}} W\left(t+\tau_{\delta}, X_{\tau_{\delta}}^{1}\right)=W(t, 1)+\widetilde{\mathrm{E}} \int_{0}^{\tau_{\delta}} W_{x}\left(t+u, X_{u}^{1}\right) d R_{u}
$$

using (4.2) and the optional sampling theorem since $W_{x}$ is bounded.

Since $\left(W\left(t+\left(s \wedge \tau_{b}\right), X_{s \wedge \tau_{b}}^{1}\right)_{0 \leq s \leq T-t}\right.$ is a martingale under $\widetilde{\mathrm{P}}$, we find that the expression on the left-hand side in (4.31) equals the first term on the right-hand side, and thus

$$
\widetilde{\mathrm{E}} \int_{0}^{\tau_{\delta}} W_{x}\left(t+u, X_{u}^{1}\right) d R_{u}=0 .
$$

On the other hand, since $W_{x}\left(t+u, X_{u}^{1}\right) d R_{u}=W_{x}(t+u, 1+) d R_{u}$ by $(3.6)$, and $W_{x}(t+u, 1+) \geq$ $\varepsilon>0$ for all $u \in\left[0, \tau_{\delta}\right]$, we see that (4.32) implies that

$$
\widetilde{\mathrm{E}} \int_{0}^{\tau_{\delta}} d R_{u}=0
$$

By (3.5) and the optional sampling theorem we see that (4.33) is equivalent to

$$
\widetilde{\mathrm{E}}\left(X_{\tau_{\delta}}^{1}\right)-1+r \widetilde{\mathrm{E}} \int_{0}^{\tau_{\delta}} X_{u}^{1} d u=0
$$

Since $X_{s} \geq 1$ for all $s \in[0, T]$ we see that (4.34) implies that $\tau_{\delta}=0 \quad \widetilde{\mathrm{P}}$-a.s. As clearly this is impossible, we see that $W_{x}(t, 1+)=0$ for all $t \in[0, T)$ as claimed (4.5).

8. It is clear that $W(t, x)$ is a function satisfying the following conditions:

$$
\begin{aligned}
& W \text { is } C^{1,2} \text { on } C \cup D, \\
& W_{t}+\mathbb{L}_{X} W \text { is locally bounded, } \\
& x \mapsto W(t, x) \text { is convex, } \\
& t \mapsto W_{x}(t, b(t) \pm) \text { is continuous }
\end{aligned}
$$

It follows that we can use the change-of-variable formula [8] for $W\left(t+s, X_{s}^{x}\right)$ :

$$
\begin{aligned}
W\left(t+s, X_{s}^{x}\right)= & W(t, x)+\int_{0}^{s}\left(W_{t}+\mathbb{L}_{X} W\right)\left(t+u, X_{u}^{x}\right) I\left(X_{u}^{x} \neq b(t+u)\right) d u \\
& +M_{s}+\int_{0}^{s} W_{x}\left(t+u, X_{u}^{x}\right) I\left(X_{u}^{x} \neq b(t+u)\right) d R_{u} \\
& +\frac{1}{2} \int_{0}^{s}\left(W_{x}\left(t+u, X_{u}^{x}+\right)-W_{x}\left(t+u, X_{u}^{x}-\right)\right) \\
& \times I\left(X_{u}^{x}=b(t+u)\right) d l_{u}^{b}(X) \\
= & W(t, x)+\int_{0}^{s}\left(G_{t}+\mathbb{L}_{X} G\right)\left(t+u, X_{u}^{x}\right) I\left(X_{u}^{x} \geq b(t+u)\right) d u+M_{s} \\
= & W(t, x)-r \int_{0}^{s} G\left(t+u, X_{u}^{x}\right) I\left(X_{u}^{x} \geq b(t+u)\right) d u+M_{s}
\end{aligned}
$$


where $M_{s}=\int_{0}^{s} W_{x}\left(u, X_{x}^{u}\right) \sigma X_{u}^{x} d \widetilde{B}_{u}$ is a martingale, $l_{u}^{b}(X)$ is the local time of $\mathrm{X}$ at the curve $b$ and we used that $G_{t}+\mathbb{L}_{X} G=-r G$ and $(3.6)+(4.2-4.5)$. Let us also note that the condition (4.37) can further be relaxed to the form where $W_{x x}=W_{1}+W_{2}$ on $C \cup D$ where $W_{1}$ is non-negative and $W_{2}$ is continuous on $[0, T) \times[1, \infty)$. This will be referred to below as the relaxed form of (4.35)-(4.38).

Inserting $s=T-t$ in (4.39), using that $W(T, x)=x-K e^{-r T}$, the sample stopping theorem and taking $\widetilde{P}$-expectation we get

$$
W(t, x)=\widetilde{\mathrm{E}}\left(X_{T-t}^{x}\right)-K \mathrm{e}^{-r T}+r \int_{0}^{T-t} \widetilde{\mathrm{E}} G\left(t+u, X_{u}^{x}\right) I\left(X_{u}^{x} \geq b(t+u)\right) d u .
$$

Inserting $x=b(t)$ in (4.40) and using (4.3) we have nonlinear integral equation for $b$ with boundary condition $b(T)=\left(K \mathrm{e}^{-r T} \vee 1\right)$ :

$$
b(t)=K\left(\mathrm{e}^{-r t}-\mathrm{e}^{-r T}\right)+F(T-t, b(t))+r \int_{t}^{T} H(t, u, b(t), b(u)) d u .
$$

Thus we have proved (4.13) and (4.14) as claimed. It remains now to show that equation (4.41) has the unique solution in the class of continuous decreasing functions satisfying $b(t)>$ $\left(K e^{-r t} \vee 1\right)$ for $0<t<T$. We provide the proof in the next section.

\section{The uniqueness of solution to the integral equation}

In order to prove the uniqueness we will follow the approach which originally was devised by Peskir for the American put option [6] and then applied to the Russian option [7].

1. We show that $b$ is the unique solution of the equation (4.41) in the class of continuous decreasing functions $c:[0, T] \rightarrow \mathbb{R}$ satisfying $c(t)>\left(K \mathrm{e}^{-r t} \vee 1\right)$ for $0 \leq t<T$. Let us assume that a function $c$ belonging to the class described above solves (4.41), and let us show that this $c$ must then coincide with the optimal stopping boundary $b$.

For this, in view of (4.40), let us introduce the function

$$
U^{c}(t, x)=\widetilde{\mathrm{E}}\left(X_{T-t}^{x}\right)-K \mathrm{e}^{-r T}+r \int_{0}^{T-t} \widetilde{\mathrm{E}} G\left(t+u, X_{u}^{x}\right) I\left(X_{u}^{x} \geq c(t+u)\right) d u
$$

for $(t, x) \in[0, T] \times[1, \infty)$. A direct inspection of the expression in (5.1) using (4.11) and (4.12) shows that $U_{x}^{c}$ is continuous on $[0, T] \times[1, \infty)$.

2. Let us define a function $W^{c}:[0, T] \times[1, \infty) \rightarrow \mathbb{R}$ by setting $W^{c}(t, x)=U^{c}(t, x)$ for $x<c(t)$ and $W^{c}(t, x)=G(t, x)$ for $x \geq c(t)$ when $0 \leq t<T$. Note that since $c$ solves (4.41) we have that $W^{c}$ is continuous on $[0, T] \times[1, \infty)$, i.e $W^{c}(t, x)=U^{c}(t, x)=G(t, x)$ for $x=c(t)$ when $0 \leq t<T$. Let $C$ and $D$ be defined by means of $c$ as in (4.8) and (4.9) respectively.

Standard arguments based on the Markov property (or a direct verification) show that $W^{c}$ i.e. $U^{c}$ is $C^{1,2}$ on $C$ and that

$$
W_{t}^{c}+\mathbb{L}_{X} W^{c}=0 \quad \text { in } C,
$$




$$
W_{x}^{c}(t, 1+)=0
$$

for all $t \in[0, T)$. Moreover, since $U_{x}^{c}$ is continuous on $[0, T) \times[1, \infty)$ we see that $W_{x}^{c}$ is continuous on $\bar{C}$. Finally, it is obvious that $W^{c}$ i.e. $G$ is $C^{1,2}$ on $D$.

3. Summarizing the preceding conclusions one can easily verify that the function $W^{c}$ satisfies (4.35)-(4.38) (in the relaxed form) so that the change-of-variable formula [8] can be applied. Using (5.3) we get

$$
\begin{gathered}
W^{c}\left(t+s, X_{s}^{x}\right)=W^{c}(t, x)+\int_{0}^{s}\left(W_{t}^{c}+\mathbb{L}_{X} W^{c}\right)\left(t+u, X_{u}^{x}\right) I\left(X_{u}^{x} \neq c(t+u)\right) d u \\
+M_{s}^{c}+\frac{1}{2} \int_{0}^{s}\left(W_{x}^{c}\left(t+u, X_{u}^{x}+\right)-W_{x}^{c}\left(t+u, X_{u}^{x}-\right)\right) \\
\times I\left(X_{u}^{x}=c(t+u)\right) d l_{u}^{c}(X)
\end{gathered}
$$

where $M^{c}$ is a martingale under $\widetilde{\mathrm{P}}$.

4. If we know that

$$
U^{c}(t, x)=G(t, x) \text { for all } x \geq c(t)
$$

holds using the general fact

$$
\left.\frac{\partial}{\partial x}\left(U^{c}(t, x)-G(t, x)\right)\right|_{x=c(t)}=W_{x}^{c}(t, c(t)-)-W_{x}^{c}(t, c(t)+)
$$

for all $0 \leq t<T$ we see that

$$
x \mapsto W^{c}(t, x) \text { is } C^{1} \text { at } c(t) \text { for each } 0 \leq t<T
$$

holds too (since $U_{x}^{c}$ is continuous).

5. To derive (5.5) first note that standard arguments based on the Markov property (or a direct verification) show that $U^{c}$ is $C^{1,2}$ on $D$ and that

$$
U_{t}^{c}+\mathbb{L}_{X} U^{c}=-r G \text { in } D .
$$

Since the function $U^{c}$ is continuous and satisfies (4.35)-(4.38) (in the relaxed form), we see that (4.39) can be applied just like in (5.4) with $U^{c}$ instead of $W^{c}$, and this yields

$$
U^{c}\left(t+s, X_{s}^{x}\right)=U^{c}(t, x)-r \int_{0}^{s} G\left(t+u, X_{u}^{x}\right) I\left(X_{u}^{x} \geq c(t+u)\right) d u+L_{s}^{c}
$$

upon using (5.2)-(5.3), (5.8) and fact that $U_{x}^{c}$ is continuous. $L^{c}$ is a martingale under $\widetilde{\mathrm{P}}$.

Next note that Ito's formula implies

$$
G\left(t+s, X_{s}^{x}\right)=G(t, x)-r \int_{0}^{s} G\left(t+u, X_{u}^{x}\right) d u+M_{s}+\int_{0}^{s} d R_{u}
$$


upon using that $G_{t}+\mathbb{L}_{X} G=-r G$ as well as that $G_{x}=1 . M$ is a martingale under $\widetilde{\mathrm{P}}$.

For $x \geq c(t)$ consider the stopping time

$$
\sigma_{c}=\inf \left\{0 \leq s \leq T-t: X_{s}^{x} \leq c(t+s)\right\} .
$$

Then using that $U^{c}(t, c(t))=G(t, c(t))$ for all $0 \leq t<T$ since $c$ solves (4.41), and that $U^{c}(T, x)=G(T, x)$ for all $x \geq 1$ by (5.1), we see that $U^{c}\left(t+\sigma_{c}, X_{\sigma_{c}}^{x}\right)=G\left(t+\sigma_{c}, X_{\sigma_{c}}^{x}\right)$. Hence from (5.9) and (5.10) using the optional sampling theorem

$$
\begin{aligned}
U^{c}(t, x)= & \widetilde{\mathrm{E}} U^{c}\left(t+\sigma_{c}, X_{\sigma_{c}}^{x}\right)+r \int_{0}^{\sigma_{c}} \widetilde{\mathrm{E}} G\left(t+u, X_{u}^{x}\right) I\left(X_{u}^{x} \geq c(t+u)\right) d u \\
= & \widetilde{\mathrm{E}} G\left(t+\sigma_{c}, X_{\sigma_{c}}^{x}\right)+r \int_{0}^{\sigma_{c}} \widetilde{\mathrm{E}} G\left(t+u, X_{u}^{x}\right) I\left(X_{u}^{x} \geq c(t+u)\right) d u \\
= & G(t, x)-r \int_{0}^{\sigma_{c}} \widetilde{\mathrm{E}} G\left(t+u, X_{u}^{x}\right) d u \\
& +r \int_{0}^{\sigma_{c}} \widetilde{\mathrm{E}} G\left(t+u, X_{u}^{x}\right) I\left(X_{u}^{x} \geq c(t+u)\right) d u \\
= & G(t, x)
\end{aligned}
$$

since $X_{u}^{x} \geq c(t+u)>1$ for all $0 \leq u \leq \sigma_{c}$. This establishes (5.5) and thus (5.7) holds too.

6. Consider the stopping time

$$
\tau_{c}=\inf \left\{0 \leq s \leq T-t: X_{s}^{x} \geq c(t+s)\right\} .
$$

Note that (5.4) using (5.2) and (5.7) reads

$$
W^{c}\left(t+s, X_{s}^{x}\right)=W^{c}(t, x)-r \int_{0}^{s} G\left(t+u, X_{u}^{x}\right) I\left(X_{u}^{x} \geq c(t+u)\right) d u+M_{s}^{c} .
$$

Using that $M^{c}$ is a martingale under $\widetilde{\mathrm{P}}$ and inserting $\tau_{c}$ in place of $s$ in (5.14), it follows upon taking the $\widetilde{\mathrm{P}}$-expectation that

$$
W^{c}(t, x)=\widetilde{\mathrm{E}} G\left(t+\tau_{c}, X_{\tau_{c}}^{x}\right)
$$

for $(t, x) \in[0, T] \times[1, \infty)$ where we use that $W^{c}(t, x)=G(t, x)$ for $x \geq c(t)$ or $t=T$. Comparing (5.15) and (4.1) we see that

$$
W^{c}(t, x) \leq W(t, x)
$$

for all $(t, x) \in[0, T] \times[1, \infty)$.

7. Let us now show that $b \geq c$ on $[0, T]$. For this, recall that by the same arguments as for $W^{c}$ we also have

$$
W\left(t+s, X_{s}^{x}\right)=W(t, x)-r \int_{0}^{s} G\left(t+u, X_{u}^{x}\right) I\left(X_{u}^{x} \geq b(t+u)\right) d u+M_{s}^{b}
$$


where $M^{b}$ is a martingale $\widetilde{\mathrm{P}}$. Fix $(t, x) \in[0, T) \times[1, \infty)$ such that $x>b(t) \vee c(t)$ and consider the stopping time

$$
\sigma_{b}=\inf \left\{0 \leq s \leq T-t: X_{s}^{x} \leq b(t+s)\right\}
$$

Inserting $\sigma_{b}$ in place of $s$ in (5.14) and (5.17) and taking the $\widetilde{\mathrm{P}}$-expectation, we get

$$
\begin{aligned}
& \widetilde{\mathrm{E}} W^{c}\left(t+\sigma_{b}, X_{\sigma_{b}}^{x}\right)=x-K \mathrm{e}^{-r t}-r \widetilde{\mathrm{E}}\left(\int_{0}^{\sigma_{b}} G\left(t+u, X_{u}^{x}\right) I\left(X_{u}^{x} \geq c(t+u)\right) d u\right) \\
& \widetilde{\mathrm{E}} W\left(t+\sigma_{b}, X_{\sigma_{b}}^{x}\right)=x-K \mathrm{e}^{-r t}-r \widetilde{\mathrm{E}}\left(\int_{0}^{\sigma_{b}} G\left(t+u, X_{u}^{x}\right) d u\right) .
\end{aligned}
$$

Hence by (5.16) we see that

$$
\widetilde{\mathrm{E}}\left(\int_{0}^{\sigma_{b}} G\left(t+u, X_{u}^{x}\right) I\left(X_{u}^{x} \geq c(t+u)\right) d u\right) \geq \widetilde{\mathrm{E}}\left(\int_{0}^{\sigma_{b}} G\left(t+u, X_{u}^{x}\right) d u\right)
$$

from where it follows by the continuity of $b$ and $c$, using $G(t, x)>0$ for $x>b(t)$, that $b(t) \geq c(t)$ for all $t \in[0, T]$.

8. Finally, let us show that $c$ must be equal to $b$. For this, assume that there is $t \in(0, T)$ such that $b(t)>c(t)$, and pick $x \in(c(t), b(t))$. Under $\widetilde{\mathrm{P}}$ consider the stopping time $\tau_{b}$ from (4.10). Inserting $\tau_{b}$ in place of $s$ in (5.14) and (5.17) and taking the $\widetilde{P}$-expectation, we get

$$
\begin{aligned}
& \widetilde{\mathrm{E}} G\left(t+\tau_{b}, X_{\tau_{b}}^{x}\right)=W^{c}(t, x)-r \widetilde{\mathrm{E}}\left(\int_{0}^{\tau_{b}} G\left(t+u, X_{u}^{x}\right) I\left(X_{u}^{x} \geq c(t+u)\right) d u\right) \\
& \widetilde{\mathrm{E}} G\left(t+\tau_{b}, X_{\tau_{b}}^{x}\right)=W(t, x) .
\end{aligned}
$$

Hence by (5.16) we see that

$$
\widetilde{\mathrm{E}}\left(\int_{0}^{\tau_{b}} G\left(t+u, X_{u}^{x}\right) I\left(X_{u}^{x} \geq c(t+u)\right) d u\right) \leq 0
$$

from where it follows by the continuity of $c$ and $b$ using $G(t, x)>0$ for $x>c(t)$ that such a point $x$ cannot exist. Thus $c$ must be equal to $b$, and the proof is complete.

\section{The arbitrage-free price and stopping region}

This section derives a representation of the arbitrage-free price for the option and the integral equation for the rational exercise boundary. We make use of the results from Sections 4 and 5, as well as the relationship (3.7) between the value functions (2.3) and (4.14).

1. After determining $W$ and the stopping boundary $b$ in (4.1) we can now solve the initial problem (2.3). Indeed, from (3.7) and (4.40) we have

$$
V(t, m, s)=s W\left(t, \frac{m \vee K}{s} ; \frac{K \mathrm{e}^{r t}}{s}\right)
$$




$$
\begin{aligned}
= & \widetilde{\mathrm{E}}\left(s X_{T-t}^{\frac{m \vee K}{s}}\right)-K \mathrm{e}^{r(t-T)} \\
& +r \int_{0}^{T-t} \widetilde{\mathrm{E}}\left(s X_{u}^{\frac{m \vee K}{s}}-K \mathrm{e}^{-r u}\right) I\left(X_{u}^{\frac{m \vee K}{s}} \geq b\left(t+u ; K \mathrm{e}^{r t} / s\right)\right) d u .
\end{aligned}
$$

2. Using that $V(t, m, s)>0$ for every $t \in[0, T)$ and $0 \leq s \leq m$ we have that

$$
K<b\left(t ; K \mathrm{e}^{r t}\right)
$$

since otherwise $V(t, 1,1)=W\left(t, K ; K \mathrm{e}^{r t}\right)=K-K \mathrm{e}^{r t} \mathrm{e}^{-r t}=0$.

Standard arguments based on the strong Markov property (see [10]) show that the stopping set in (2.3) is given by

$$
\begin{aligned}
D & =\left\{(t, m, s): V(t, m, s)=(m-K)^{+}\right\} \\
& =\left\{(t, m, s): s W\left(t, \frac{m \vee K}{s} ; \frac{K \mathrm{e}^{r t}}{s}\right)=(m-K)^{+}\right\} \\
& =\left\{(t, m, s): W\left(t, \frac{m \vee K}{s} ; \frac{K \mathrm{e}^{r t}}{s}\right)=\frac{m \vee K}{s}-\frac{K \mathrm{e}^{r t}}{s} \mathrm{e}^{-r t}\right\} \\
& =\left\{(t, m, s): \frac{m \vee K}{s} \geq b\left(t ; \frac{K \mathrm{e}^{r t}}{s}\right)\right\} \\
& =\left\{(t, m, s): m \geq s b\left(t ; \frac{K \mathrm{e}^{r t}}{s}\right)\right\}
\end{aligned}
$$

for $0 \leq t<T$ and $0<s \leq m$ where in final equality we used (6.2).

Thus the optimal stopping time in (2.3) is given by

$$
\tau_{g}=\inf \left\{0 \leq u<T-t: M_{u} \geq g\left(t+u, S_{u}\right)\right\}
$$

where the function $g$ is given by

$$
g(t, s)=s b\left(t ; \frac{K \mathrm{e}^{r t}}{s}\right) .
$$

3. We now show some properties of the optimal stopping boundary $g$. From (6.5) one can see that in order to compute $s \rightarrow g(t, s)$ for fixed $t \in[0, T)$ we need to calculate the optimal stopping boundary $b\left(\cdot ; \frac{K \mathrm{e}^{r t}}{s}\right)$ with a scaling strike as a solution to (4.41) for every $s>0$. Using that the problem (2.3) time-homogeneous, in the sense that the gain function does not depend on time, it follows that $t \mapsto g(t, s)$ is decreasing on $[0, T)$ for each $s>0$ fixed. It is also clear from $(2.3)$ that $s \mapsto g(t, s)$ is increasing on $[0, \infty)$ with $g(t, 0+)=K$ for $t \in[0, T)$ fixed. From (4.41) we see that $K \mapsto b(t ; K)$ is continuous on $(0, \infty)$ for each $t \in[0, T)$ fixed. Hence we have that $s \mapsto g(t, s)$ is continuous on $(0, \infty)$ for $t \in[0, T)$ fixed. From (6.5) it is easily to seen that $g(t, s) \uparrow s b_{0}(t)$ as $s \rightarrow \infty$ for fixed $t \in[0, T)$ where $b_{0}(t)=b(t ; 0)$ is the optimal stopping boundary for the Russian option [7]. The Figure 2 illustrates computer drawing of the optimal stopping boundaries $s \mapsto g(u, s)$ and $s \mapsto g(v, s)$ for $0<u<v<T$. The boundaries shift to the right as time goes to $T$ and the optimal stopping time is the first hitting time of $M$ over $g(\cdot, S)$. 


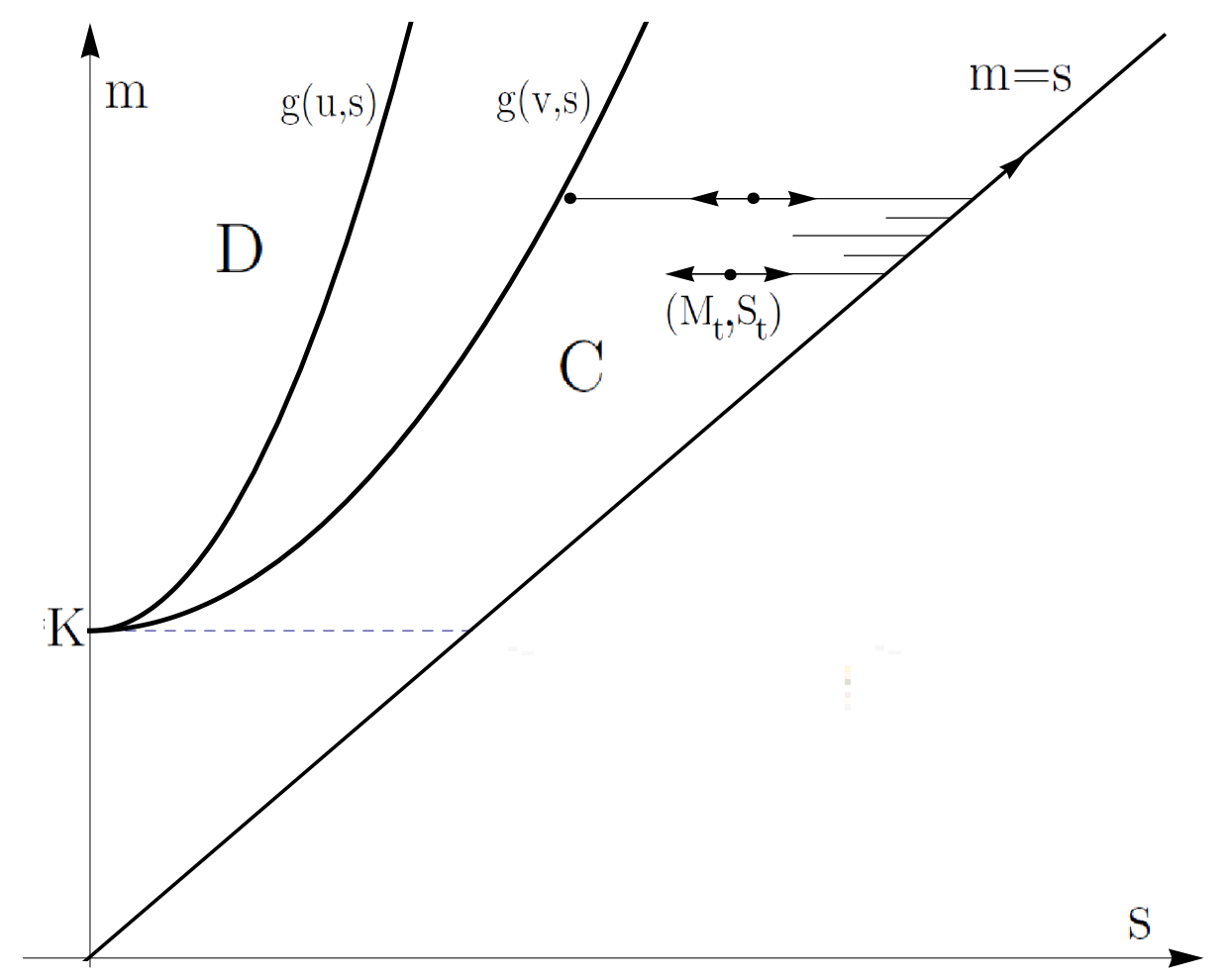

Figure 2. A computer drawing of the optimal stopping boundaries $s \mapsto g(u, s)$ and $s \mapsto g(v, s)$ for $0<u<v<T$.

\section{Conclusion}

In this section we make a conclusion of the results obtained in this paper and propose the future research program.

The main idea of the paper is to show a reduction of the three-dimensional optimal stopping problem (2.3) to the two-dimensional optimal stopping problem (4.1). However, after solving the two-dimensional problem and coming back to the initial problem we vary parameter in (4.1), and thus the problem remains inherently three-dimensional. We determined expressions for the arbitrage-free price (6.1) and the rational exercise boundary (6.5).

As remarked in the introduction, the reduction of the three-dimensional problem to a twodimensional one with a scaling strike can be used to tackle the optimal stopping problem for the British lookback option with fixed strike. Performing the financial analysis of American and British versions of this option as in [2] leads to an extensive programme of research which we aim to present in a subsequent publication.

The method of scaling strike also allows to examine the problem (2.3) in exponential Lévy models and then after reducing dimensions and applying the local time-space calculus for general semimartingales (see [9]) the nonlinear integral equations for optimal stopping boundaries can be obtained.

Acknowledgements. The author is grateful to Professor G. Peskir for fruitful discussions and hints concerning the reduction of the three-dimensional problem to two dimensions. 


\section{References}

[1] Gapeev, P. (2006). Discounted optimal stopping for maxima in diffusion models with finite horizon. Electron. J. Probab. 11 (1031-1048).

[2] Glover, K., Peskir, G. and Samee, F. (2011). The British Russian option. Stochastics $80(315-332)$.

[3] Kyprianou, A. and Отт, C. (2012). A capped optimal stopping problem for the maimum process. arXiv:1204.3119v1 [math.PR]

[4] Pedersen, J. L. (2000). Discounted optimal stopping problems for the maximum process. J. Appl. Probab. 37 (972-983).

[5] Peskir, G. (1998). Optimal stopping of the maximum process: The maximality principle. Ann. Probab. 26 (1614-1640).

[6] Peskir, G. (2005). On the American option problem. Math. Finance 15 (169-181).

[7] Peskir, G. (2005). The Russian option: Finite horizon. Finance Stoch. 9 (251-267).

[8] Peskir, G. (2005). A change-of-variable formula with local time on curves. J. Theoret. Probab. 18 (499-535).

[9] Peskir, G. (2007). A change-of-variable formula with local time on surfaces. Sém. de Probab. XL, Lecture Notes in Math. 1899, Springer (69-96).

[10] Peskir, G. and Shiryaev, A. N. (2006). Optimal Stopping and Free-Boundary Problems. Lectures in Mathematics, ETH Zürich, Birkhäuser.

[11] Shepp, L. and Shiryaev, A. N. (1993). The Russian option: Reduced regret. Ann. Appl. Probab. 3 (631-640).

[12] Shepp, L. and Shiryaev, A. N. (1994). A new look at the Russian option. Theory Probab. Appl. 39 (103-119).

[13] Shiryaev, A. N. (1999). Essentials of Stochastic Finance. World Scientific.

Yerkin Kitapbayev

School of Mathematics

The University of Manchester

Oxford Road

Manchester M13 9PL

United Kingdom

Yerkin.Kitapbayev@postgrad.manchester.ac.uk 